\title{
Quantitative Models for Germination and Infection of Pseudoperonospora cubensis in Response to Temperature and Duration of Leaf Wetness
}

\author{
L. F. Arauz, K. N. Neufeld, A. L. Lloyd, and P. S. Ojiambo
}

First, second, and fourth authors: Department of Plant Pathology, and third author: Department of Mathematics, North Carolina State University, Raleigh 27695.

Current address of L. F. Arauz: University of Costa Rica, San Jose, 2060, Costa Rica. Accepted for publication 12 May 2010.

\begin{abstract}
Arauz, L. F., Neufeld, K. N., Lloyd, A. L., and Ojiambo, P. S. 2010. Quantitative models for germination and infection of Pseudoperonospora cubensis in response to temperature and duration of leaf wetness. Phytopathology 100:959-967.

The influence of temperature and leaf wetness duration on germination of sporangia and infection of cantaloupe leaves by Pseudoperonospora cubensis was examined in three independent controlled-environment experiments by inoculating plants with a spore suspension and exposing them to a range of leaf wetness durations ( 2 to $24 \mathrm{~h}$ ) at six fixed temperatures $\left(5\right.$ to $\left.30^{\circ} \mathrm{C}\right)$. Germination of sporangia was assessed at the end of each wetness period and infection was evaluated from assessments of disease severity 5 days after inoculation. Three response surface models based on modified forms of the Weibull function were evaluated for their ability to describe germination of sporangia and infection in response to temperature and leaf wetness duration. The models estimated 15.7 to 17.3 and 19.5 to $21.7^{\circ} \mathrm{C}$ as the optimum temperature $(t)$ range for germination

was observed at $t=20^{\circ} \mathrm{C}$ but broader optimum curves resulted from wetness periods $>8 \mathrm{~h}$. Model 1 of the form $f(w, t)=f(t) \times(1-\exp \{-[B \times$ $\left.w]^{D}\right\}$ ) resulted in smaller asymptotic standard errors and yielded higher correlations between observed and predicted germination and infection data than either model 2 of the form $f(w, t)=A\left(1-\exp \left\{-[f(t) \times(w-C)]^{D}\right\}\right)$ or model 3 of the form $f(w, t)=\left[1-\exp \left\{-(B \times w)^{2}\right\}\right] / \cosh [(t-F) G / 2]$. Models 1 and 2 had nonsignificant lack-of-fit test statistics for both germination and infection data, whereas a lack-of-fit test was significant for model 3. The models accounted for $\approx 87 \%$ (model 3) to $98 \%$ (model 1 ) of the total variation in the germination and infection data. In the validation of the models using data generated with a different isolate of $P$. cubensis, slopes of the regression line between observed and predicted germination and infection data were not significantly different $(P>0.2487)$ and correlation coefficients between observed and predicted values were high $\left(r^{2}>0.81\right)$. Models 1 and 2 were used to construct risk threshold charts that can be used to estimate the potential risk for infection based on observed or forecasted temperature and leaf wetness duration.
\end{abstract} and infection, respectively, with little germination or infection at 5 or $30^{\circ} \mathrm{C}$. For wetness periods of 4 to $8 \mathrm{~h}$, a distinct optimum for infection
Additional keywords: cucurbit downy mildew, disease forecasting.
Cucurbit downy mildew, caused by the oomycete Pseudoperonospora cubensis, is considered the most damaging disease of cucurbitaceous crops worldwide. In the eastern United States and California, cucurbit downy mildew is an annual problem on cucurbits in late summer and fall. Since the 1960s, resistance in cucumber had adequately controlled the disease with minimal use of fungicides (15). However, in 2004, the cucumber crop in North Carolina, Virginia, Delaware, Maryland, and New Jersey was devastated by downy mildew and regional losses of the crop were estimated as $40 \%$ (15). This resurgence of downy mildew was attributed partly to new and highly aggressive strains of $P$. cubensis (8). Presently, in the United States and elsewhere, host plant resistance in cucurbits is no longer sufficient to control the disease (22). Thus, management of the disease now relies heavily on the application of fungicides.

Weather variables, particularly temperature and leaf wetness, greatly influence the outbreaks of cucurbit downy mildew epidemics. Unlike germination of sporangia, the effects of individual weather variables on disease development have been investigated extensively. The optimum temperature for infection has been reported to be 15 to $20^{\circ} \mathrm{C}$ (7). The minimum duration of leaf wetness needed for infection to occur is $2 \mathrm{~h}(6)$. In the absence of leaf

Corresponding author: P. S. Ojiambo; E-mail address: peter_ojiambo@ncsu.edu

doi:10.1094/PHYTO-100-9-0959

(c) 2010 The American Phytopathological Society wetness, the effect of other weather variables on disease development is very limited (27). Yang et al. (36) reported that a daily difference in temperature of $\leq 5^{\circ} \mathrm{C}$, daily mean relative humidity $(\mathrm{RH}) \geq 80 \%$, and daily mean temperature of 15 to $25^{\circ} \mathrm{C}$ were the thresholds for infection in autumn and, based on their results, an early warning model was developed to predict occurrence of downy mildew in unheated greenhouses in China. The combined effects of temperature and leaf wetness on infection parameters (germination and infection) of $P$. cubensis have not been extensively studied. Current knowledge of the interacting effects of temperature and leaf wetness on infection of $P$. cubensis is mainly based on a study by Cohen (6), who observed that the minimum, maximum, and optimum levels for symptom production were dependent on either temperature, duration of leaf wetness, or both factors. Based on the latter observation, a qualitative description of the interacting effects of temperature and leaf wetness duration was proposed to describe disease development under field conditions (6).

The combined effects of temperature and wetness duration on a variety of diseases $(3,10,11,24)$ have been quantitatively described using polynomial equations whose many parameters lack a clear epidemiological significance. Nonlinear models for evaluating the response of foliar parasites to combined effects of temperature and wetness duration have been proposed by Duthie (9). Theoretically, nonlinear models provide a more parsimonious description of empirical data than models that wholly or in part comprise polynomial equations (5). Although parameters of the nonlinear 
models proposed by Duthie (9) can be interpreted to provide information on the mechanisms involved in the disease response, only a few reports $(4,12,35)$ have evaluated these models using empirical data. In addition, there are no reports that quantitatively examine the relative performance of these models using empirical data to assess their usefulness in describing the combined effects of temperature and leaf wetness on infection parameters of plant pathogens.

In the United States, a downy mildew forecasting system is available (26) and provides growers with information on the risk of disease outbreak during the growing season. This advisory program is based on, among other factors, pathogen sporulation in response to temperature and duration of leaf wetness at the inoculum source and along the projected pathway of sporangia transport. Infection is predicted and a spray is recommended when the risk of disease outbreak is medium to high. The disease forecasting system issues the risk of downy mildew outbreak based on, among other factors, a qualitative assessment of temperature and duration of leaf wetness and may not be accurate for a wide range of temperature and leaf wetness combinations.

A quantitative analysis of the effect of weather variables is useful in developing mathematical models that allow for prediction of disease outbreak under a wide range of weather conditions. Such epidemiological models that predict specific phases of pathogen development can provide useful knowledge for managing plant diseases (18). However, quantitative models describing the response of infection parameters of $P$. cubensis to temperature and duration of leaf wetness are lacking. Once developed, the predictors can be used in combination with the current forecasting tools to generate more accurate forecasts for initial infection along the projected pathway of sporangia transport. This is particularly important for cucurbit downy mildew (which spreads rapidly under favorable weather conditions), for which growers are much more concerned with "whether" infection will occur rather than "how much" infection will actually take place. Thus, the objectives of this study were to (i) characterize combinations of temperature and leaf wetness duration favorable for germination and infection of $P$. cubensis and (ii) develop models for quantifying the effect of temperature and leaf wetness durations on the infection parameters of $P$. cubensis.

\section{MATERIALS AND METHODS}

Plant growth conditions. Cantaloupe plants (cv. Kermit) were grown in circular, $8.0-\mathrm{cm}$ Styrofoam cups (one plant/cup) in vermiculite and maintained in a growth chamber under the following conditions: 12-h photoperiod; photon flux density $\approx 370 \mu \mathrm{mol} \mathrm{m} \mathrm{m}^{-2} \mathrm{~s}^{-1}$ at plant height, provided by cool white fluorescent tubes and incandescent bulbs; temperatures of 24 to $28^{\circ} \mathrm{C}$. The plants were watered twice daily with half-strength Hoagland's solution (14) and once daily with deionized water.

Inoculation and incubation. $P$. cubensis, isolate SC-07, which was isolated from cantaloupe in Sampson County, NC, in 2007 and maintained on frozen infected leaves at $-80^{\circ} \mathrm{C}$, was used to produce inoculum as described by Lebeda (20). Prior to inoculation, randomly selected plants at the two-true-leaf stage $(\approx 21$ days old) were first preconditioned overnight at one of six temperature regimes $\left(5,10,15,20,25\right.$, and $\left.30^{\circ} \mathrm{C}\right)$. A spore suspension $\left(1 \times 10^{3}\right.$ sporangia $\mathrm{ml}^{-1}$ in deionized water $)$ was then sprayed onto the abaxial side of the true leaves to incipient run-off using a Preval mist sprayer (Complete Unit 267' Precision Valve Corporation, Yonkers, NY). Immediately after inoculation, the plants were enclosed in plastic bags to ensure that leaf wetness persisted for the required periods and incubated at the temperatures mentioned above in darkened growth chambers.

In order to evaluate germination of sporangia over different wetness periods, plants were removed from each chamber after $2,4,8,12$, and $24 \mathrm{~h}$, and 10 -mm leaf discs were taken from the second leaf of each plant. The leaf discs were immediately fixed and decolorized in acetic acid/ethanol for $48 \mathrm{~h}$ (21) and then stained with cotton blue in lactophenol and covered with a cover slip. Nongerminated and germinated sporangia were heavily stained and appeared light blue, respectively, while plant tissues remained white. Sporangia of $P$. cubensis do not germinate directly by the formation of a germ tube but germinate indirectly by zoospore release through cytoplasmic cleavage. Thus, a sporangium was considered to have germinated when its cytoplasmic content was emptied. At least 50 sporangia per treatment were evaluated microscopically for germination on the host tissue. Germination rates of $P$. cubensis sporangia are high and variation in germination from different batches of inoculum is minimal (17).

Environmental requirements for infection were characterized in two separate stages. First, under each of the above temperature regimes (the order of temperature treatments was assigned at random), plants were exposed to five different wetness periods by removing three randomly selected plants from each growth chamber after 2, 4, 8, 12, and $24 \mathrm{~h}$. After drying the leaves with fan-forced air for 10 to $15 \mathrm{~min}$, the plants were returned to their respective growth chambers and exposed to ambient humidity. Temperatures in the growth chambers were maintained within a 0.5 to $1.5^{\circ} \mathrm{C}$ difference of the test value, while the $\mathrm{RH}$ was dependent on the temperature and ranged between 40 and $60 \%$. In the second stage, all plants were removed from the growth chambers after $24 \mathrm{~h}$ and subjected to standard incubation conditions ( 21 and $18^{\circ} \mathrm{C}, 12$ - and 12 -h day and night temperatures and light regimes; 75 to $90 \%$ RH) in a Percival incubator (Model I-36VL, Percival Scientific Inc., Perry, IA). Disease severity was visually assessed 5 days after inoculation as percentage of leaf area with chlorotic and necrotic symptoms. Data from the two true leaves on a plant per treatment were averaged for each leaf wetness period. Diseased leaf area was considered to be a valid measure for infection, because the plants were subjected to standardized conditions after the initial 24-h infection period, and local lesions of downy mildews generally do not expand significantly after symptom appearance (29). Both the germination and infection experiments were conducted three times and there were three replicate plants with two inoculated leaves per plant for each combination of temperature and leaf wetness duration.

Data analysis and model development. In the first set of analyses, means for each response variable taken over the three plants for each experiment were subjected to analysis of variance to determine the effects of temperature $(t)$ and leaf wetness duration $(w)$ and their interaction $(t \times w)$ on germination of sporangia and infection. The GLM procedure of SAS (version 9.1; SAS Institute, Cary, NC) was used for this analysis with the experiment as a blocking factor. There were no significant differences between the three runs of the experiment for either germination or infection data; therefore, all subsequent analyses were performed on data averaged over the three runs.

The combined effects of temperature $(t)$ and leaf wetness duration $(w)$ on a given infection parameter of $P$. cubensis, $f(w, t)$, were further evaluated using two nonlinear equations of the form

$$
f(w, t)=f(t) \times\left(1-\exp \left\{-[B(w-C)]^{D}\right\}\right)
$$

and

$$
f(w, t)=A\left(1-\exp \left\{-[f(t) \times(w-C)]^{D}\right\}\right)
$$

Unless stated otherwise, italicized lower- and uppercase letters represent variables and parameters, respectively. A typographical error appears in equations 3.1 and 3.2 of Duthie (9), in which the expression $\{-[B(w-C)]\}$ and $\{-[f(t) \times(w-C)]\}$ rather than $[B(w-C)]$ and $[f(t) \times(w-C)]$, respectively, are raised to $D$. The expression $f(t)$, which is the upper asymptote in equation 1 and 
the intrinsic rate in equation 2, is determined by $t$ and is given by the equation

$$
f(t)=y_{t}=E^{\prime}\{\exp [(t-F) G /(H+1)]\} /\{1+\exp [(t-F) G]\}
$$

in which

$$
E^{\prime}=E[(H+1) / H] H^{1 /(H+1)}
$$

Briefly, equations 1 and 2 are modified forms of a Weibull function (34) that describe the monotonic increase of $f(w, t)$ with respect to $w$ from a minimum of $f(w, t)=0$ at $w=C$ to a temperature-dependent upper limit when $w$ is large. Thus, $C(C>0)$ characterizes the lag period before the response of $f(w, t)$ to $w$ begins. The parameter $A$ characterizes the upper limit on $f(w, t)$ as $w$ is extended indefinitely. The parameter $B(0<B<1)$ characterizes the intrinsic rate of increase of $f(w, t)$ with respect to $w$ and while the parameter $D(D>0)$ is the fraction of wetness duration in which $f(w, t)$ decelerates (9). In equation $1, y_{t}\left(y_{t}>0\right)$ characterizes the scale of the response to $w$ and describes the unimodal response of $y_{t}$ to $t$ at each $w$. The parameter $E(E>0)$, characterizes the scale of the response to $t$, whereas the parameters $F(-\infty<F<+\infty)$ and $G(G>0)$ characterize the intrinsic rate of change of $f(w, t)$ with respect to the optimal temperature and $t$, respectively. At $t=G, f(w, t)$ approaches an upper limit of $E$ when $w$ is large. As $t$ deviates from $G$ at each $w, f(w, t)$ declines at an intrinsic rate of $G$ toward a lower limit of 0 . The parameter $H$ characterizes asymmetry in the response to $t$. The optimum temperature is given by the equation $t_{\text {opt }}=F-(1 / G) \log (H)$ (9).

Disregarding asymmetry (i.e., $H=1$ and $E^{\prime}=2 E$ ) and assuming that the pathogen responds immediately to a period of wetness (i.e., $C=0$ ), equation 2.1 can be rewritten as

$$
f(t)=y_{t}=2 E /\{\exp [-(t-F) G / 2]+\exp [(t-F) G / 2]\}
$$

$$
=E \times \operatorname{sech}[(t-F) G / 2]=E / \cosh [(t-F) G / 2]
$$

in which sech and cosh are the hyperbolic secant and cosine, respectively (see Appendix for derivation). If germination and infection are measured on a scale of 0 to 1 , the value of $E$ in equation 1 can be fixed to $E=1$. Thus, equation 1 above reduces to a four-parameter model of the form:

$$
f(w, t)=\left[1-\exp \left\{-(B \times w)^{D}\right\}\right] / \cosh [(t-F) G / 2]
$$

Nonlinear models (equations 1, 2, and 3, henceforth referred to as models 1,2 , and 3 , respectively) were fitted to data by an iterative and derivative free nonlinear regression approach using the NLIN procedure of SAS. Based on preliminary regression analysis, model 3 was reduced to a three-parameter model of the form:

$$
f(w, t)=\left[1-\exp \left\{-(B \times w)^{2}\right\}\right] / \cosh [(t-F) G / 2]
$$

Thus, the final nonlinear estimates for model 3 were based on a three-parameter equation where the value of $D(=1.428$ and 1.337 for germination and infection, respectively) was fixed to 2 . Goodness-of-fit was evaluated by the magnitude of asymptotic confidence intervals on parameter estimates and by inspection of observed and predicted values plotted simultaneously against $w$ or $t$. Heteroscedasticity was evaluated by inspecting standardized residual errors plotted against predicted values. Overparameterization was assessed by the magnitudes of asymptotic standard errors and asymptotic correlation coefficients. Because the coefficient of determination $\left(R^{2}\right)$ is not valid for nonlinear least squares approximations, evaluation of regression models was based on the simple correlation $\left(r_{\hat{y} y}\right)$ between observed $(y)$ and predicted $(\hat{y})$ germination or infection levels, the lack-of-fit (LOF) $F$ statistic (37), and the significance of estimated parameters. Regressions were based on germination and infection data averaged over the replications rather than on pooled data to obtain appropriate LOF estimates. The LOF $F$ statistic is not valid for nonlinear models but at least gives an indication about the suitability of the models because, even for nonlinear models, the variance ratio for the LOF statistic is approximately $F$ distributed when the sample size is large (31). Following model validation (see below), isopaths derived from models 1 and 2 for the range of temperature and leaf wetness durations required to attain thresholds values of the response variables (germination of sporangia or infection) were used to generate charts for predicting the risk of cucurbit downy mildew development based on observed or forecasted temperature and leaf wetness duration.

Model validation. To validate the models, an independent set of data on germination of sporangia and infection was generated as described above using a different isolate of $P$. cubensis, isolate JC-09. Isolate JC-09 was isolated from cucumber in Johnston County, NC, in 2009 and maintained as described above. The observed germination and infection values were compared with values predicted by the models. Validation tests were first analyzed by linear regression of observed $(y)$ versus predicted $(\hat{y})$ values and the differences in the slopes for the observed and predicted data were tested using PROC GLM in SAS. The second validation test utilized the coefficient of variation of the residuals of errors (16), $C V_{R E}$, which is calculated as

$$
C V_{R E}=\left[\sum(y-\hat{y})^{2}\right]^{1 / 2} /\left(n \hat{y}_{m}\right)
$$

where $n$ is the number of comparisons and $\hat{y}_{m}$ is the average of predicted values across all the comparisons of temperature and durations of leaf wetness.

\section{RESULTS}

Temperature and duration of leaf wetness significantly $(P<$ $0.0001)$ affected germination of sporangia and infection of cantaloupe leaves by $P$. cubensis (Table 1). Further, germination and infection were also responsive to interacting effects of temperature and leaf wetness duration (Table 1). No differences in germination of sporangia and infection were observed between

\begin{tabular}{|c|c|c|c|c|c|c|}
\hline \multirow[b]{2}{*}{ Source } & \multicolumn{3}{|c|}{ Germination } & \multicolumn{3}{|c|}{ Infection } \\
\hline & $\mathrm{df}$ & SS & $P>F$ & $\mathrm{df}$ & SS & $P>F$ \\
\hline Experiment & 2 & 15.12 & 0.4350 & 2 & 20.05 & 0.3613 \\
\hline Temperature $(t)$ & 5 & $8,140.87$ & 0.0001 & 5 & $3,498.86$ & 0.0001 \\
\hline$t \times w$ & 20 & $1,358.59$ & 0.0001 & 20 & $1,099.76$ & 0.0001 \\
\hline
\end{tabular}
the three independent experiments and, thus, data were pooled over experiments for final analysis and presentation.

Germination of sporangia on cantaloupe leaves showed a broad optimum within the temperature range of 10 to $25^{\circ} \mathrm{C}$ at $w \geq 8 \mathrm{~h}$

TABLE 1. Analysis of variance for the effects of temperature and duration of leaf wetness on sporangia germination and infection of cantaloupe by Pseudoperonospora cubensis 
(Fig. 1A). The highest proportion of germinated sporangia (>0.55) was attained at $t=10$ to $15^{\circ} \mathrm{C}$ for $w \geq 12 \mathrm{~h}$, at $t=20^{\circ} \mathrm{C}$ for $w \geq 8 \mathrm{~h}$, and at $t=25^{\circ} \mathrm{C}$ at $w=12 \mathrm{~h}$. The proportion of germinated sporangia at 5 or $30^{\circ} \mathrm{C}$ was $\approx 0.18$. An increase in infection resulted from increasing leaf wetness duration at all temperatures evaluated (Fig. 1B). Infection levels $>0.25$ were obtained for $w=24 \mathrm{~h}$ at $t=10^{\circ} \mathrm{C}, w \geq 12 \mathrm{~h}$ at $t=15^{\circ} \mathrm{C}$, and $w$ $\geq 8 \mathrm{~h}$ for $t=20$ to $25^{\circ} \mathrm{C}$. Very minimal infection was observed at $w=2 \mathrm{~h}$ and $t=5$ to $10^{\circ} \mathrm{C}$. For wetness periods of 4 to $8 \mathrm{~h}$, a distinct optimum for infection was observed at $t=20^{\circ} \mathrm{C}$ but broader optimum curves resulted from $w>8 \mathrm{~h}$. A comparison of the observed response surfaces (Fig. 1A and B) indicated that terminal levels of germination of sporangia and infection were not very different, although initial germination rates were higher than initial infection rates.

Although the three nonlinear models resulted in significant $(P<$ $0.0001)$ fit to sporangia germination and infection data, they varied in their ability to predict the combined effects of temperature and leaf wetness duration on the infection parameters of $P$. cubensis. In the first set of the analysis, parameter estimates in model 1 were significantly $(P<0.05)$ different from zero for germination and infection data, except for parameter $C$, which was not significantly different from zero $(P>0.05)$. Thus, the model was refitted to respective germination and infection data as a six-parameter model without the parameter $C$ to generate final estimates and their corresponding statistics. All the models pre-
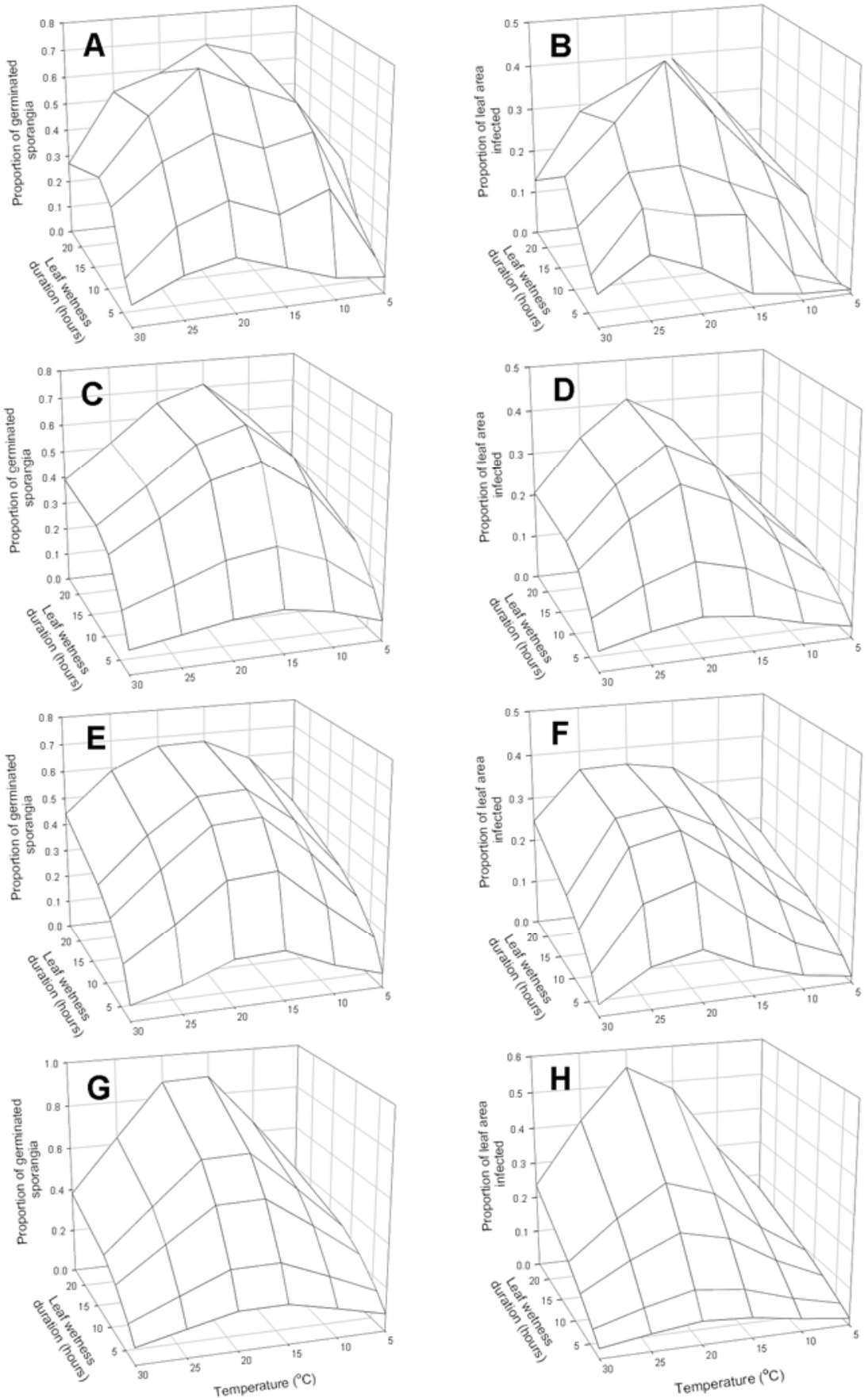

Fig. 1. Effects of temperature $(t)$ and duration of leaf wetness $(w)$ on infection parameters of Pseudoperonospora cubensis (isolate SC-07) sporangia on cantaloupe: A, Observed germination of sporangia and $\mathbf{B}$, proportion of leaf area infected 5 days after inoculation. Predicted germination of sporangia based on $\mathbf{C}$, model 1: $f(w, t)=f(t) \times\left(1-\exp \left\{-[B \times w]^{D}\right\}\right) ; \mathbf{E}$, model 2: $f(w, t)=A\left(1-\exp \left\{-[f(t) \times(w-C)]^{D}\right\}\right)$; and $\mathbf{G}$, model 3: $f(w, t)=\left[1-\exp \left\{-(B \times w)^{2}\right\}\right] / \cosh [(t-F) G / 2]$. Predicted proportion of leaf area infected 5 days after inoculation based on $\mathbf{D}$, model $1: f(w, t)=f(t) \times\left(1-\exp \left\{-[B \times w]^{D}\right\}\right) ; \mathbf{F}, \operatorname{model} 2: f(w, t)=A\{1-\exp [-f(t) \times$ $\left.(w-C)]^{D}\right\}$; and $\mathbf{H}$, model 3: $f(w, t)=\left[1-\exp \left\{-(B \times w)^{2}\right\}\right] / \cosh [(t-F) G / 2]$. 
dicted a unimodal response of germination and infection to temperature, with germination and infection being greatest near the middle of the temperature range and least near the extreme temperatures (Fig. 1C to $\mathrm{H}$ ).

Model 1 generated parameter estimates with asymptotic standard errors that were much smaller for germination than for infection data, except for parameter $G$ (Table 2). Response surfaces for both germination and infection as predicted by model 1 closely resembled response surfaces for the observed data (Fig. $1 C$ and $\mathrm{D}$ ). Estimates for parameters $B, D$, and $E$, were relatively larger for sporangia germination than for infection, whereas parameter estimates for $F, G$, and $H$ were relatively smaller for sporangia germination than for infection data (Table 2). For both germination and infection data, the parameter $D$ was $>1$, indicating that the absolute germination and infection rates in response to leaf wetness duration first increased and then decreased thereafter over the range of our experimental conditions. Model 1, describing germination and infection by $P$. cubensis, yielded correlation coefficients $r_{\hat{y} y}$ of 0.941 and 0.923 , respectively (Table 3). An $F$ test performed at $\alpha=0.01$ did not indicate a significant LOF between predictions from model 1 and observed sporangia germination and infection (Table 3). The germination and infection curves describing the asymptotic parameter in response to temperature attained their optima at 15.7 and $19.5^{\circ} \mathrm{C}$, respectively (Fig. 2A). A contour plot showed a faster increase in germination and infection with increasing temperature than increasing duration of leaf wetness (Fig. 3); however, this increase was more rapid for germination than infection.

Model 2 resulted in parameters that were all significantly $(P<$ $0.05)$ different from zero for both germination and infection data (Table 4). Unlike for germination (Fig. 1E), the response surface for infection as predicted by model 2 did not closely resemble the response surface for observed infection (Fig. 1F). Parameter estimates for infection were larger than those for sporangia germination infection, except for parameters $A$ and $C$. The corresponding asymptotic standard errors of the parameters were much smaller for germination than for infection except for parameters $A$ and $F$ (Table 4). Unlike for model 1 , the parameter $D$ for model 2 was $<1$, indicating that absolute germination and infection rates in response to leaf wetness duration decreased over the entire range of our experimental conditions. Based on this model, the calculated optimum temperatures for germination and infection were 17.0 and $21.7^{\circ} \mathrm{C}$, respectively. Maximum values for the rate parameter were observed at these optimum temperatures with the rate parameter $\omega[=f(t)]$ for infection being $5 \%$ higher than for germination (Fig. 2B). Model 2 yielded correlation coefficients $r_{\hat{y} y}$ of 0.934 and 0.919 for germination and infection, respectively (Table 3). An LOF $F$ test performed for model 1 was not significant at $\alpha=0.01$ for sporangia germination and infection data (Table 3). Contour plots for model 2 also showed a faster increase in germination and infection with increasing temperature than with increasing leaf wetness duration (Fig. 4), and this increase was also more rapid for germination than for infection.

Parameter estimates for model 3 were all significantly $(P<$ $0.001)$ different from zero for sporangia germination and infection data (Table 5). However, the response surfaces predicted by this model slightly resembled the response surface for observed germination but not infection (Fig. $1 \mathrm{G}$ and $\mathrm{H}$ ). Parameter estimates were much larger for infection than for germination data except for the parameter $B$. Asymptotic standard errors of estimates were smaller for germination than for infection, except for the parameter $B$ (Table 5). Unlike models 1 and 2 , an $F$ test performed at $\alpha=0.01$ indicated a significant LOF between the model 3 and germination and infection data (Table 3). Values for the parameter $F$ (equivalent to the optimum temperature based on model 3) were 17.3 and $19.0^{\circ} \mathrm{C}$ for germination and infection, respectively. Optimum temperatures for germination and infection based on this model were slightly higher than for germination and infection based on models 1 and 2, respectively.

In the validation test for model 1 using a different isolate of $P$. cubensis (JC-09), the regression line for observed versus predicted values (Fig. 5) was not significantly $(P=0.5260)$ different

TABLE 2. Parameter estimates of model $1, f(w, t)=f(t) \times\left(1-\exp \left\{-[B \times w]^{D}\right\}\right)$, describing germination and infection of cantaloupe by Pseudoperonospora cubensis based on the combined effects of temperature and duration of leaf wetness ${ }^{\mathrm{a}}$

\begin{tabular}{|c|c|c|c|c|c|c|c|c|}
\hline \multirow[b]{2}{*}{ Parameter } & \multicolumn{4}{|c|}{ Sporangia germination } & \multicolumn{4}{|c|}{ Infection } \\
\hline & Estimate & Asymptotic SE & Asymptotic $C I_{L}$ & Asymptotic $C I_{U}$ & Estimate & Asymptotic SE & Asymptotic $C I_{L}$ & Asymptotic $C I_{U}$ \\
\hline$B$ & 0.193 & 0.010 & 0.172 & 0.214 & 0.179 & 0.016 & 0.147 & 0.212 \\
\hline$D$ & 1.428 & 0.116 & 1.197 & 1.658 & 1.337 & 0.165 & 1.009 & 1.665 \\
\hline$F$ & 11.176 & 1.295 & 8.600 & 13.751 & 19.024 & 2.011 & 15.039 & 23.009 \\
\hline$G$ & 0.248 & 0.021 & 0.204 & 0.291 & 0.256 & 0.014 & 0.227 & 0.285 \\
\hline$H$ & 0.328 & 0.094 & 0.139 & 0.515 & 0.893 & 0.335 & 0.229 & 1.556 \\
\hline
\end{tabular}

a In the model, $f(t)=E^{\prime}\{\exp [(t-F) G /(H+1)]\} /\{1+\exp [(t-F) G]\}$, in which $E^{\prime}=E[(H+1) / H] H^{1 /(H+1)}$. The parameters $B, D, E, F, G$ and $H$ are as defined in the main text; $t=$ temperature and $w=$ leaf wetness duration. $\mathrm{SE}$ is the standard error and $C I_{L}$ and $C I_{U}=$ lower and upper limits of the $95 \%$ confidence interval around the parameter estimates.

TABLE 3. Comparison of three models describing combined effects of temperature and leaf wetness duration on sporangia germination and infection on cantaloupe 5 days after inoculation with Pseudoperonospora cubensis

\begin{tabular}{|c|c|c|c|c|c|}
\hline Model $^{\mathrm{a}}$ & Number of parameters & $r_{\hat{Y Y}} \mathrm{~b}$ & Approximate $R^{2}$ value & LOF- $F^{\mathrm{c}}$ & $\mathrm{df}^{\mathrm{d}}$ \\
\hline \multicolumn{6}{|c|}{ Germination } \\
\hline Model 1 & 6 & 0.941 & 0.981 & 0.504 & 24,60 \\
\hline Model 2 & 7 & 0.934 & 0.937 & 1.503 & 23,60 \\
\hline \multicolumn{6}{|l|}{ Infection } \\
\hline Model 1 & 6 & 0.923 & 0.970 & 1.579 & 24,60 \\
\hline Model 2 & 7 & 0.919 & 0.938 & 1.213 & 23,60 \\
\hline Model 3 & 3 & 0.834 & 0.867 & 5.756 & 27,60 \\
\hline
\end{tabular}

a Model 1: $f(w, t)=f(t) \times\left(1-\exp \left\{-[B \times w]^{D}\right\}\right)$, model 2: $f(w, t)=A\left(1-\exp \left\{-[f(t) \times(w-C)]^{D}\right\}\right)$, and model 3: $f(w, t)=\left[1-\exp \left\{-(B \times w)^{2}\right\}\right] / \cosh [(t-F) G / 2]$.

Parameters in the models are defined in the main text, while $t=$ temperature and $w=$ leaf wetness duration.

${ }^{\mathrm{b}}$ Simple correlation between observed and predicted germination of infection.

${ }^{c}$ Lack-of-fit (LOF) $F$ statistic.

${ }^{\mathrm{d}}$ Degrees of freedom for LOF $F$ ratio. 
from 1 (i.e., the slope of the observed = predicted line) and the adjusted $r^{2}$ was 0.90 and 0.81 for sporangia germination and infection, respectively. The regression lines between the observed and predicted values based on model 2 were also not significantly different $(P=0.2487)$, albeit with slightly lower adjusted $r^{2}$ values of 0.87 and 0.78 for germination and infection, respectively (data not shown). The coefficient of variation of the residual of errors $\left(C V_{R E}\right)$ for sporangia germination was 1.2 and $2.1 \%$ for model 1 and 2 , respectively. The $C V_{R E}$ values for infection data were 7.8 and $9.2 \%$ for models 1 and 2, respectively. Validation tests for model 3 were not performed due to the significant LOF test statistics.

\section{DISCUSSION}

Prior to this study, the combined effects of temperature and moisture on germination of $P$. cubensis sporangia had not been reported. Further, previous studies on ecological and epidemiological aspects of cucurbit downy mildew either examined temperature and moisture effects on infection separately $(7,27)$ or did not quantify (6) the relationship of temperature and moisture with infection. Nonetheless, results from these previous studies on infection of cucurbits by $P$. cubensis are still useful for comparison. Cohen and Rotem (7) reported that the optimum temperature for infection occurred at 15 to $20^{\circ} \mathrm{C}$, while an optimum of 15 to $25^{\circ} \mathrm{C}$ was reported by Yang et al. (36). Cohen (6) also reported a broader range $\left(5\right.$ to $\left.20^{\circ} \mathrm{C}\right)$ of optimum temperature
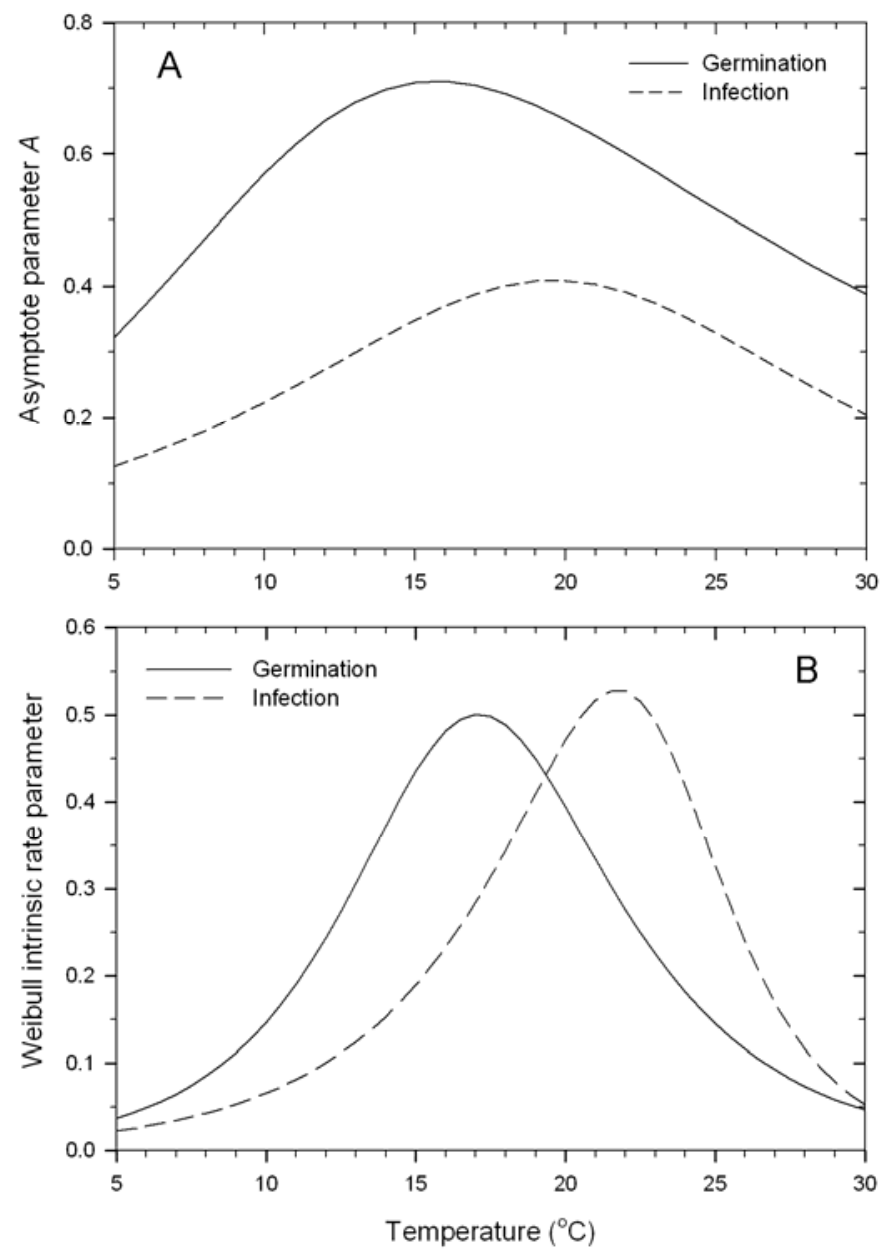

Fig. 2. A, Asymptote parameter and $\mathbf{B}$, the Weibull intrinsic rate parameter as a functions of temperature based on model 1: $f(w, t)=f(t) \times(1-\exp \{-[B \times$ $\left.\left.w]^{D}\right\}\right)$ and model 2: $f(w, t)=A\left(1-\exp \left\{-[f(t) \times(w-C)]^{D}\right\}\right)$, respectively, for predicting sporangia germination and proportion of leaf area infected 5 days after inoculation with Pseudoperonospora cubensis. that was dependent on dew period. The optimum temperature for infection were 20,10 to 20 , and 5 to $20^{\circ} \mathrm{C}$ at dew periods of 2,12 , and $24 \mathrm{~h}$, respectively (6). Our results revealed a similar trend, with an optimum of 10 to $25^{\circ} \mathrm{C}$ for both germination and infection. However, we observed germination and infection at $30^{\circ} \mathrm{C}$, unlike in the study by Cohen (6), where the maximum temperature under which infection occurred was $28^{\circ} \mathrm{C}$. This discrepancy may be due to differences in the strains of $P$. cubensis. Highly aggressive strains of $P$. cubensis were linked to the resurgence of downy mildew in 2004 in the United States (8). Thus, it is likely that these new strains have a slightly higher maximum temperature for infection than older strains of $P$. cubensis.

Various quantitative models have been proposed to describe spore germination and host infection in response to temperature and duration of leaf wetness. The most commonly used mathematical functions are the polynomial models $(2,23,30)$. The flexibility of polynomial curves, mathematical simplicity of polynomial functions, and availability of curve-fitting methods has made polynomial models a common choice to describe the response of infection parameters to temperature and moisture $(5,19)$. Nonetheless, polynomial functions have little biological meaning and their fit is limited by the degree of polynomial that can be used (33). A major drawback with the implementation of polynomials in predictive models is the potential for unrealistic results if the equations are applied to temperatures and wetness durations outside the range used to derive the parameter estimates. Duthie (9) proposed modified versions of the Weibull
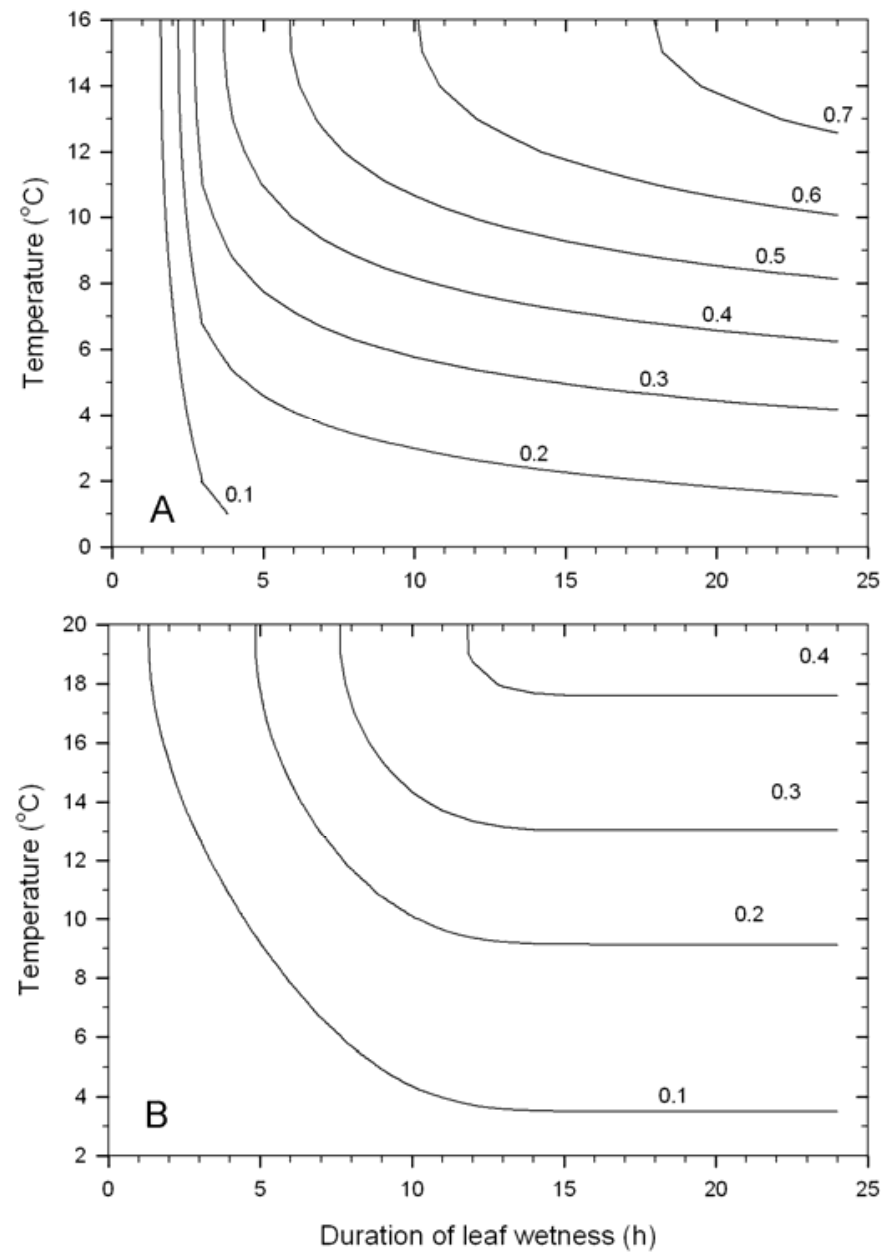

Fig. 3. Contour plots of proportions of $\mathbf{A}$, germination and $\mathbf{B}$, infection potentials for different combinations of temperature and duration of leaf wetness as predicted by model 1: $f(w, t)=f(t) \times\left(1-\exp \left\{-[B \times w]^{D}\right\}\right)$. Each line (isopath) depicts a contour line connecting points of equal proportions of germination or infection. 
equation (34) to model response of foliar parasites to both temperature and wetness duration. One main advantage of these models over other nonlinear models is that all the parameters used to describe the response to temperature and wetness duration have some biological significance $(9,28)$. In this study, these modified versions of the Weibull equations provided a good description of germination and infection of cantaloupe inoculated by $P$. cubensis under the controlled conditions. Our results also showed a clear relationship between the asymptote parameters and temperature and this allowed us to indirectly evaluate the effect of temperature on germination of sporangia and infection through its effect on the model parameters.

Although the models proposed by Duthie (9) have been used to model the response of specific pathogens to combined effects of temperature and leaf wetness duration $(4,12,35)$, a systematic evaluation of these models based on empirical data had not been reported previously. In this study, the combined effects of temperature and leaf wetness duration on sporangia germination and infection by $P$. cubensis were best described by model 1 . Although model 2 provided good fit to germination data, the model did not provide an equally good fit to infection data. Asymptotic standard errors of parameter estimates for infection data were larger for model 2 than for model 1. Further, parameter estimates for infection data were relatively more highly correlated for model 2 than for model 1 (data not shown). Parameters that are highly correlated and have large confidence intervals indicate an overparameterization of the model (13). Model 3, which is a reduced form of model 1 where asymmetry is disregarded, did not provide a good fit to either germination or infection data, as indicated by the significant LOF test statistics. The goodness-offit of model 3 may be improved by estimating the asymmetry parameter $H$ with a more detailed data set rather than fixing it to 1. Because sporangia germination and infection were measured on a scale of 0 to 1 , the value of $E$ was fixed to 1 in model 3, although the actual values for maximum germination and infection were $<1$. A similar assumption is also implied by forms of the monomolecular, Gompertz, or logistic equation that lack an explicit upper asymptote parameter. Fixing of the upper limit to 1 when the actual upper limit is $<1$ has been shown to result in inadequate performance of models (25).

The response of sporangia germination and infection to temperature was unimodal with an optimum temperature of 15.7 to 17.3 and 19.5 to $21.7^{\circ} \mathrm{C}$, respectively. Germination and infection increased with wetness duration in a sigmoidal fashion. The interaction of temperature and wetness duration also affected spore germination and infection. For example, fewer hours of leaf wetness duration were required to achieve a specific level of germination or infection as temperature approached an optimum. Our results also showed that temperature, rather than duration of leaf wetness, was the dominant factor that determined the rate and extent of infection by $P$. cubensis. This is in agreement with reports by Yang et al. (36) but in contrast to the study by Cohen
(6), in which leaf wetness duration was considered to be the dominant factor affecting the extent of infection. Differences in the analytical approaches may explain the discrepancy with regard to this observation. Whereas we used a quantitative approach to reach this conclusion, Cohen (6) based his conclusion on a qualitative description of the effects of temperature and leaf wetness duration on infection. Given our model, the duration of leaf wetness can be viewed as the factor that allows germination or infection to occur, while temperature determines the progress of these infection parameters. Our results can be coupled with a sporulation model (i.e., amount of sporangia produced and the proportion that escapes the canopy) to facilitate the development of a quantitative forecast system. Currently, a sporulation model
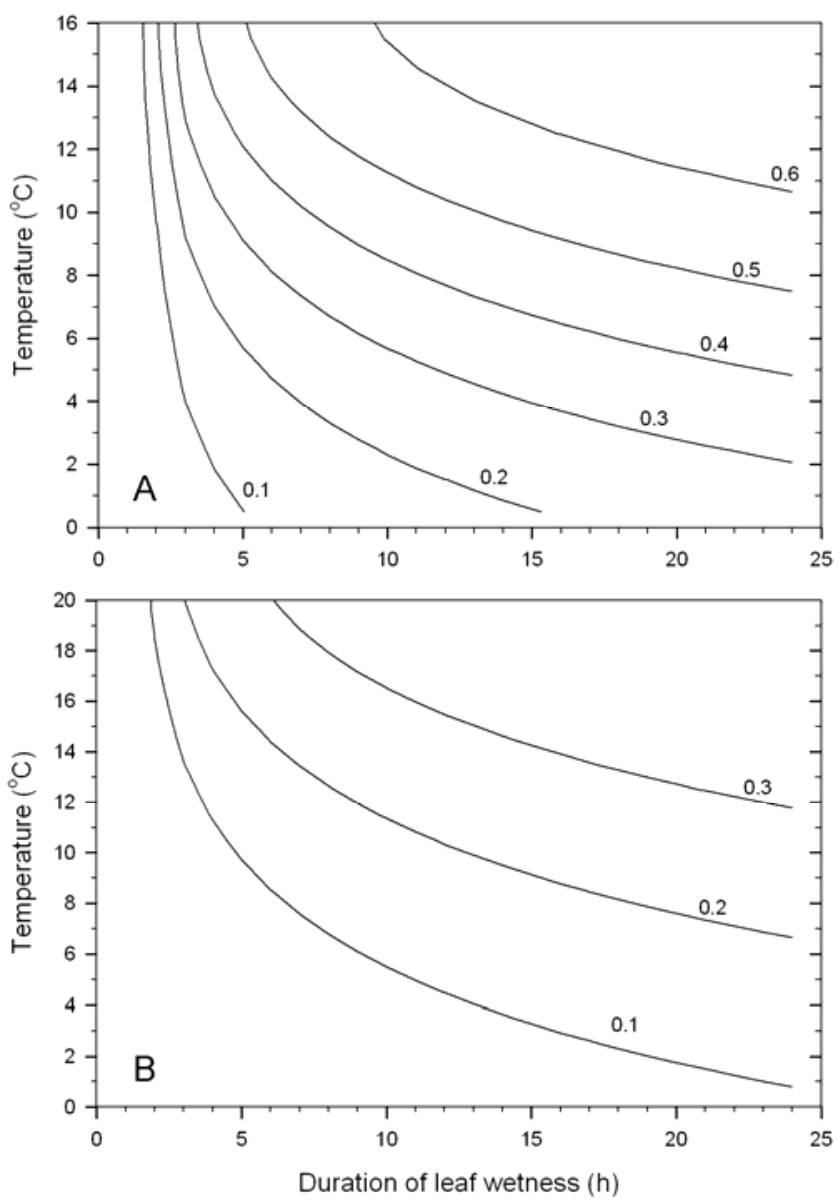

Fig. 4. Contour plots of proportions of $\mathbf{A}$, germination and $\mathbf{B}$, infection potentials for different combinations of temperature and durations of leaf wetness as predicted by model 2: $f(w, t)=A\left(1-\exp \left\{-[f(t) \times(w-C)]^{D}\right\}\right)$. Each line (isopath) depicts a contour line connecting points of equal proportions of germination or infection.

TABLE 4. Parameter estimates of model 2, $f(w, t)=A\left(1-\exp \left\{-[f(t) \times(w-C)]^{D}\right\}\right)$, describing germination and infection of cantaloupe by Pseudoperonospora cubensis based on the combined effects of temperature and duration of leaf wetness ${ }^{\mathrm{a}}$

\begin{tabular}{|c|c|c|c|c|c|c|c|c|}
\hline \multirow[b]{2}{*}{ Parameter } & \multicolumn{4}{|c|}{ Sporangia germination } & \multicolumn{4}{|c|}{ Infection } \\
\hline & Estimate & Asymptotic SE & Asymptotic $C I_{L}$ & Asymptotic $C I_{U}$ & Estimate & Asymptotic SE & Asymptotic $C I_{L}$ & Asymptotic $C I_{U}$ \\
\hline$A$ & 0.689 & 0.045 & 0.598 & 0.779 & 0.362 & 0.020 & 0.322 & 0.401 \\
\hline$C$ & 1.725 & 0.161 & 1.404 & 2.045 & 1.391 & 0.386 & 0.625 & 2.157 \\
\hline$E$ & 0.501 & 0.155 & 0.192 & 0.809 & 0.529 & 0.188 & 0.156 & 0.901 \\
\hline$F$ & 16.716 & 1.128 & 14.472 & 18.959 & 22.802 & 0.974 & 20.872 & 24.732 \\
\hline$G$ & 0.511 & 0.052 & 0.406 & 0.616 & 0.621 & 0.089 & 0.444 & 0.798 \\
\hline
\end{tabular}

${ }^{a}$ In the model, $f(t)=E^{\prime}\{\exp [(t-F) G /(H+1)]\} /\{1+\exp [(t-F) G]\}$, in which $E^{\prime}=E[(H+1) / H] H^{1 /(H+1)}$. The parameters $A, C, D, E, F, G$, and $H$ are as defined in the main text, while $t=$ temperature and $w=$ leaf wetness duration. SE is the standard error and $C I_{L}$ and $C I_{U}=$ lower and upper limits, respectively, of the $95 \%$ confidence interval around the parameter estimates. 
is not available and we are in the process of developing such a model for $P$. cubensis. Once developed, the sporulation model could be used to estimate the number of sporangia produced per unit lesion area in a field based on environmental conditions. In a subsequent infection period, the proportion of sporangia that escapes the canopy and is available for aerial transport and capable of causing disease can then be calculated. Any loss of viability of sporangia between sporulation and infection can be accounted for using a survival model (17). The quantitative nature of such as forecasting system should, in theory, allow for more accurate predictions than a similar but qualitative system currently being used for cucurbit downy mildew.

The models developed in this study were not validated using field data, and a systematic field validation of the models is needed before the models can be used for predictive purposes in the field. As indicated by Jørgensen and Bendoricchio (16), the amount of data available for any modeling project varies greatly and the availability of data on the real system will determine the validation process. In this study, the ideal method of validation would require placing inoculated plants in a disease-free field to control the source of inoculum. However, due to the mode of spread of $P$. cubensis, introducing inoculated plants in the field when the disease is absent is discouraged in North Carolina. Given this constraint to field validation, we are exploring other approaches that can still be used to validate the models using field data. In addition, the models developed in this study are not intended for direct application in cucurbit downy mildew forecasting because they do not account for temperature differences during intermittent periods of leaf wetness that occur in the field. However, the results of these models may still be used to improve the current weather-based advisory system. For example, model 1 can be used in conjunction with the FLEXPART plume dispersal model (32), which is used to predict spore transport, to forecast initial infection along the projected pathway of spore transport, based on observed or forecasted temperature and leaf wetness periods along the inoculum trajectory. Inevitably, temperature and leaf wetness durations will vary along any given spore trajectory and the models developed in this study could facilitate the delineation of areas with similar or different risks of disease outbreak. Further, cantaloupe, a relatively less susceptible host compared with other cucurbit crop hosts such as cucumber, was used in the present study. Wu et al. (35) observed an interaction of host resistance and wetness period whereby a longer wetness period was required to achieve a specific level of disease for a partially resistant compared with a susceptible host. Thus, additional studies are recommended to determine how host resistance would influence the interactive effects of temperature and leaf wetness duration on infection by $P$. cubensis. The inclusion of host susceptibility and interrupted leaf wetness would increase the predictive capacity of the model.

\section{APPENDIX}

Derivation of equation 3. From equation 1

$$
f(w, t)=f(t) \times\left(1-\exp \left\{-[B(w-C)]^{D}\right\}\right)
$$

in which

$$
f(t)=y_{t}=E^{\prime}\left[\frac{\exp [(t-F) G /(H+1)]}{1+\exp [(t-F) G]}\right]
$$

and where

$$
E^{\prime}=E[(H+1) / H] H^{1 /(H+1)}
$$

If symmetry is disregarded, then $H=1$ and substituting $H=1$ in equation $\mathrm{A} 2.1$ results in

$$
E^{\prime}=2 E
$$

Thus, it follows that equation A2 can be rewritten as
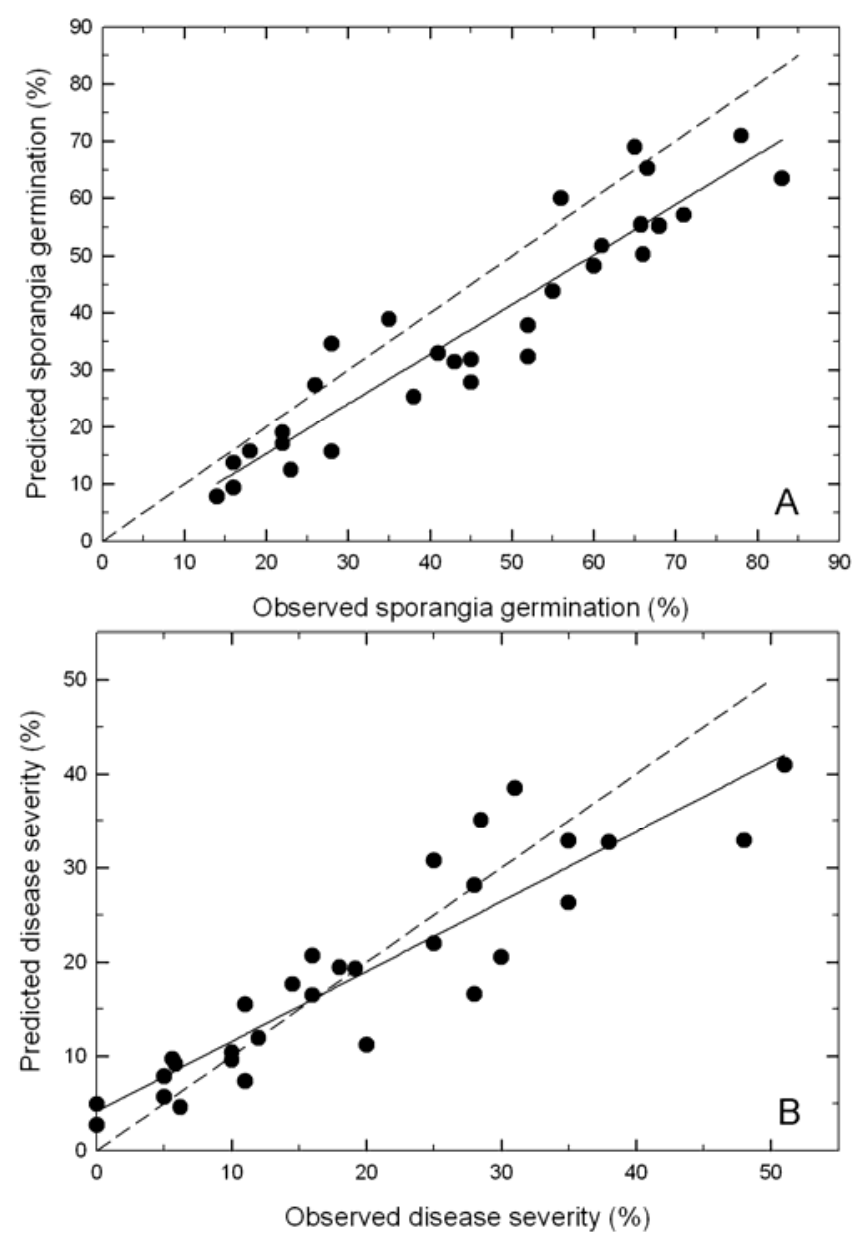

Fig. 5. Validation of model 1: $f(w, t)=f(t) \times\left(1-\exp \left\{-[B \times w]^{D}\right\}\right)$ for describing the combined effects of temperature and leaf wetness duration on sporangia germination and infection of cantaloupe inoculated with Pseudoperonospora cubensis isolate JC-09. Comparison between observed versus predicted $\mathbf{A}$, sporangia germination and $\mathbf{B}$, leaf area infected. The regression line (solid line) is not significantly different from observed $=$ modeled (dashed line) and the orrelation coefficient (adjusted $\left.r^{2}\right)=0.90$ and

\begin{tabular}{|c|c|c|c|c|c|c|c|c|}
\hline \multirow[b]{2}{*}{ Parameter } & \multicolumn{4}{|c|}{ Sporangia germination } & \multicolumn{4}{|c|}{ Infection } \\
\hline & Estimate & Asymptotic SE & Asymptotic $C I_{L}$ & Asymptotic $C I_{U}$ & Estimate & Asymptotic SE & Asymptotic $C I_{L}$ & Asymptotic $C I_{U}$ \\
\hline$B$ & 0.053 & 0.003 & 0.046 & 0.059 & 0.017 & 0.001 & 0.014 & 0.019 \\
\hline$F$ & 17.361 & 0.338 & 16.688 & 18.033 & 19.061 & 0.489 & 18.093 & 20.029 \\
\hline
\end{tabular}
0.81 for germination of sporangia and infection, respectively.

TABLE 5. Parameter estimates for the combined effects of temperature and duration of leaf wetness based on model $3, f(w, t)=\left[1-\exp \left\{-(B \times w)^{2}\right\}\right] / \cosh [(t-$ $F) G / 2$ ], describing germination and infection of cantaloupe by Pseudoperonospora cubensis ${ }^{\mathrm{a}}$

a Parameters $B, F$, and $G$ are as defined in the main text; $t=$ temperature and $w=$ leaf wetness duration. SE is the standard error and $C I_{L}$ and $C I_{U}=$ lower and upper limits, respectively, of the $95 \%$ confidence interval around the parameter estimates. 


$$
f(t)=y_{t}=E\left[\frac{2 \cdot \exp [(t-F) G / 2]}{1+\exp [(t-F) G]}\right]
$$

Dividing the denominator and numerator by $\exp [(t-F) G / 2]$, and given that $\exp (z / 2) / \exp (z)=\exp (-z / 2)$, equation $\mathrm{A} 3$ can be rewritten as

$$
f(t)=y_{t}=E\left[\frac{2}{\exp [-(t-F) G / 2]+\exp [(t-F) G / 2]}\right]
$$

By definition (1), the hyperbolic secant of a function $z$ is expressed as

$$
\operatorname{sech}(z)=[1 / \cosh (z)]=2 /[\exp (z)+\exp (-z)]
$$

Where $\cosh (z)$ is the hyperbolic cosine of $z$. If, in equation A4, $(t-F) G / 2=z$ and using the definition of a hyperbolic function, equation $\mathrm{A} 4$ can rewritten as

$$
f(t)=y_{t}=E \times \operatorname{sech}[(t-F) / 2]=E / \cosh [(t-F) G / 2]
$$

If $C=0, E=1$, and $y_{t}$ is expressed as in equationA6, then it follows that equation A1 can be expressed as

$$
f(w, t)=\left[1-\exp \left\{-(B \times w)^{D}\right\}\right] / \cosh [(t-F) G / 2]
$$

\section{ACKNOWLEDGMENTS}

This research was supported in part by a grant from the United States Department of Agriculture Pest Information Platform for Extension and Education program. We thank H. Scherm, University of Georgia, for his advice on some aspects of this study.

\section{LITERATURE CITED}

1. Abramowitz, M., and Stegun, I. A. 1964. Handbook of mathematical functions (with formulas, graphs, and mathematical tables). U.S. Government Printing Office, Washington, DC.

2. Arauz, L. F., and Sutton, T. B. 1989. Temperature and wetness duration requirements for apple infection by Botryosphaeria obtusa. Phytopathology 79:440-444.

3. Arny, C. J., and Rowe, R. C. 1991. Effects of temperature and duration of surface wetness on spore production and infection of cucumbers by Didymella bryoniae. Phytopathology 81:206-209.

4. Carisse, O., Bourgeois, G., and Duthie, J. A. 2000. Influence of temperature and leaf wetness duration on infection of strawberry leaves by Mycosphaerella fragariae. Phytopathology 90:1120-1125.

5. Carisse, O., and Kushalappa, A. P. 1990. Development of an infection model for Cercospora carotae on carrot based on temperature and leaf wetness duration. Phytopathology 80:1233-1238.

6. Cohen, Y. 1977. The combined effects of temperature, leaf wetness, and inoculum concentration on infection of cucumbers with Pseudoperonospora cubensis. Can. J. Bot. 55:1478-1487.

7. Cohen, Y., and Rotem, J. 1969. The effects of lesion development, air temperature, and duration of moist period on sporulation of Pseudoperonospora cubensis in cucumbers. Israel J. Bot. 18:135-140.

8. Colucci, S. J. 2008. Host range, fungicide resistance and management of Pseudoperonospora cubensis, causal agent of cucurbit downy mildew. M.S. thesis, North Carolina State University, Raleigh.

9. Duthie, J. A. 1997. Models of the response of foliar parasites to the combined effects of temperature and duration of wetness. Phytopathology 87:1088-1095.

10. Elliot, V. J. 1988. Response models for conidiospore germination and germ tube elongation of Mycosphaerella fragariae as influenced by temperature and moisture. Phytopathology 78:645-650.

11. Evans, K. J., Nyquist, W. E., and Latin, R. X. 1992. A model based on temperature and leaf wetness duration for establishment of Alternaria leaf blight of muskmelon. Phytopathology 82:890-895.
12. Furuya, H., Takanashi, H., Fuji, S., Nagai, Y., and Naito, H. 2009. Modeling infection of spring onion by Puccinia allii in response to temperature and leaf wetness. Phytopathology 99:951-956.

13. Hau, B., Eisensmith, S. P., and Kranz, J. 1985. Construction of temporal models. Pages 31-65 in: Advances in Plant Pathology. Vol. 3, Mathematical Modelling of Crop Disease. C. A. Gilligan, ed. Academic Press, New York.

14. Hoagland, D. R., and Arnon, D. I. 1950. The water culture method for growing plants without soil. Calif. Agric. Exp. Stn. Circ. 347:1-23.

15. Holmes, G., Wehner, T., and Thornton, A. 2006. An old enemy reemerges. Am. Veg. Grower. February:14-15.

16. Jørgensen, S. E., and Bendoricchio, G. 2001. Fundamentals of Ecological Modelling, 3rd ed. Elsevier Sciences, Ltd., Oxford.

17. Kanetis, L., Holmes, G. J., and Ojiambo, P. S. 2010. Survival of Pseudoperonospora cubensis sporangia exposed to solar radiation. Plant Pathol. 59:313-323.

18. Kushalappa, A. C. 2001. BREMCAST: Development of a system to forecast risk levels of downy mildew on lettuce (Bremia lactuace). Int. J. Pest Manage. 47:1-5.

19. Lalancette, N., Ellis, M. A., and Madden, L. V. 1988. Development of an infection efficiency model for Plasmopara viticola on American grape based on temperature and duration of leaf wetness. Phytopathology 78:794-800.

20. Lebeda, A. 1986. Pseudoperonospora cubensis. Pages 81-85 in: Methods of Testing Vegetable Crops for Resistance to Plant Pathogens. A. Lebeda, ed. Sempra Research Institute of Vegetable Crops, Czech Republic.

21. Lebeda, A., and Reinink, K. 1991. Variation in the early development of Bremia lactucae on lettuce with different levels of field resistance. Plant Pathol. 40:232-237.

22. Lebeda, A., and Widrlechner, M. P. 2003. A set of cucurbitaceae taxa for differentiation of Pseudoperonospora cubensis pathotypes. J. Plant Dis. Prot. 110:337-349.

23. Lui, L. H., and Kushalappa, A. C. 2003. Models to predict potato tuber infection by Pythium ultimum from duration of wetness and temperature, and leak-lesion expansion from storage duration and temperature. Postharvest Biol. Technol. 27:313-322.

24. Monroe, J. S., Santini, J. B., and Latin, R. 1997. A model defining the relationship between temperature and leaf wetness duration, and infection of watermelon by Colletotrichum orbiculare. Plant Dis. 81:739-742.

25. Neher, D. A., and Campbell, C. L. 1992. Underestimation of disease progress rates with logistic, monomolecular and Gompertz models when maximum disease intensity is less than 100 percent. Phytopathology 82:811-814.

26. Ojiambo, P. S., Kanetis, L., and Holmes, G. J. 2009. Forecasting long distance movement of Pseudoperonospora cubensis and the Cucurbit ipmPIPE. (Abstr.) Phytopathology 99:S171.

27. Palti, J., and Cohen, Y. 1980. Downy mildew of cucurbits (Pseudoperonospora cubensis): The fungus and its hosts, distribution, epidemiology and control. Phytoparasitica 8:109-147.

28. Pennypacker, S. P., Knobe, H. D., Antle, C. E., and Madden, L. V. 1980. A flexible model for studying plant disease progression. Phytopathology 70:232-235.

29. Populer, C. 1981. Epidemiology of downy mildews. Pages 57-105 in: The Downy Mildews. D. M. Spencer, ed. Academic Press, New York.

30. Scherm, H., and van Bruggen, A. H. C. 1993. Response surface models for germination and infection of Bremia lactucae, the fungus causing downy mildew of lettuce. Ecol. Model. 65:281-296.

31. Schnute, J. 1981. A versatile growth model with statistically stable parameters. Can. J. Fish. Aquat. Sci. 38:1128-1140.

32. Stohl, A., Forster, C., Frank, A., Seibert, P., and Wotawa, G. 2005. Technical note: The Lagrangian particle dispersion model FLEXPART version 6.2. Atmos. Chem. Phys. Discuss. 5:4739-4799.

33. Venus, J. C., and Causton, D. R. 1979. Plant growth analysis: The use of Richards function as an alternative to polynomial exponential. Ann. Bot. 43:623-632.

34. Weibull, W. 1951. A statistical distribution of wide applicability. J. Appl. Mech. 18:293-297.

35. Wu, L., Damicone, J. P., Duthie, J. A., and Melouk, H. A. 1999. Effects of temperature and wetness duration on infection of peanut cultivars by Cercospora arachidicola. Phytopathology 89:653-659.

36. Yang, X., Li, M., Zhang, Z., and Hou, Y. 2007. Early warning model for cucumber downy mildew in unheated greenhouses. N. Z. J. Agric. Res. 50:1261-1268.

37. Zwietering, M. H., de Koos, J. T., de Wit, J. C., and van't Riet, K. 1991. Modeling of bacterial growth as a function of temperature. Appl. Environ. Microbiol. 57:1094-1101. 